\title{
Ferromagnetism in Hubbard Models: Low Density Route
}

\author{
E. Müller-Hartmann \\ Institut für Theoretische Physik, Universität zu Köln, Zülpicher Str. 77, 50937 Köln, \\ Germany
}

Thirty years ago the Hubbard model was introduced by Gutzwiller, Hubbard and Kanamori with the main purpose of mimicking the ferromagnetism of transition metals. Soon after, Nagaoka and Thouless pointed out a basic mechanism for ferromagnetism in strongly correlated electron systems by studying the motion of a single hole in a half-filled Hubbard model. This important work was hoped to shed light onto metallic ferromagnetism from the low doping regime. Unfortunately, this low doping route towards ferromagnetism has not been successful as far as rigorous results for finite doping concentrations are concerned. In the work presented here, we start from the opposite limit of low particle concentrations. In this limit we provide the first proof of a fully polarized metallic ground state for a Hubbard model. The proof proceeds by mapping Hubbard "zigzag" chains onto a continuum model with an additional degree of freedom and local first Hund's rule coupling. For this model the maximum total spin multiplet is shown to be the unique ground state for infinite Hubbard coupling. Our proof may open a low density route towards the understanding of the ferromagnetism of Hubbard models.

PACS numbers: 71.2\%. $+a, 75.10 . L p$

The Hubbard model was introduced about 30 years agd 1 . 3 mainly for the purpose of mimicking the ferromagnetism of transition metals (see also the the paper by R. Strack and D. Vollhardt in this volume). In view of this it is quite astonishing that up to this point our understanding of the ferromagnetism of Hubbard models is very limited. All the rigorous results proving the presence of a ground state with net polarization 4 , 6 are either concerned with half-filled bands (i.e. with ferromagnetism of insulators) or with somewhat artificial flat-band systems which have ferromagnetic ground states even for vanishing interaction strength. Very recently Tasaki succeeded in proving local stability of the ferromagnetic ground state for nearly-flat-band systems There is not a single rigorous result which would provide a positive answer towards the basic question which was in the minds of the fathers 1 目 3 of the Hubbard model:

- Is a strong intra-atomic Coulomb repulsion sufficient for pushing a partially 


\section{E. Müller-Hartmann}

filled (i.e. paramagnetic) band into a fully polarized ferromagnetic ground state (Nagaoka state)?

Nagaoka's theorem 19 was, undoubtedly, an early important contribution to the subject. It tells us the precise conditions for the formation of ferromagnetic polarons around electrons or holes doped into a half-filled Hubbard model at infinite coupling strength_see also 10, 11.6). The puzzling results obtained for two and more electrons or holes 12 made obvious, though, that the problem of the global alignment of ferromagnetic polarons, in case they existed, is much harder to solve. In fact, not a single rigorous result establishing a ferromagnetic ground state for finite doping concentration is known. The low doping route towards metallic ferromagnetism which was so impressively opened by Nagaoka's theorem has failed as far as rigorous results are concerned.

There are, however, several pieces of evidence in favor or in disfavor of ferromagnetism for Hubbard models on various lattices. Let us first look at the hypercubic lattices of dimensions $d=1$ to $d=\infty$. For the linear chain $(d=1)$ it is exactly known that there is never a ferromagnetic ground state, except for the limit of infinite coupling strength where due to complete spin degeneracy the fully polarized states are among the many ground states 13 In the limit of infinite dimension a variational argument using Gutzwiller wave functions also proves that the ground state is never fully polarized, not even for infinite coupling.14 Here, partially polarized ground states have not been excluded and probably exist, but have not been established so far because the numerical solution of the mean-field theory at $d=\infty$ (see the paper by A. Georges in this volume) is hard in the very strong coupling limit. Variational estimates of the regime of stability of a Nagaoka ground state suggest that the lower the dimension of the lattice the larger this regime is, if it exists. Therefore, this problem has been studied most extensively for the square lattice. The variational proofs of instability of the Nagaoka state for the square lattice leave a rather small regime of possible stability which can be characterized by a critical electron or hole concentration (relative to half-filling) of $\delta_{c r}=0.29$ and by a critical coppling of $U_{c r}=63 t$ where $t$ denotes the nearest neighbor hopping amplitude. 15. 16. 17, 18 The estimates of the ground state energy deduced from a high temperature expansion 19 allow for a critical density $\delta_{c r}$ (again relative to half-filling) of at most 0.10 and are consistent with $\delta_{c r}=0$. This result appears not in agreement with finite square plaquette Lanczos data20 and with densitymatrix renormalization group (DMRG) extrapolations from multi-chain systems 21 which both suggest a $\delta_{c r}$ of about 0.20 . The evidence in the case of the square lattice seems still controversial and not conclusive. The even more favorable case of the double chain has not peen studied as extensively as the square lattice. Lanczos data 20 and DMRG data21 agree on $\delta_{c r}=0.22$ for the double chain. It would be very helpful if a high temperature expansion for the double chain also became available.

In the presence of so much uncertainty in the case of hypercubic lattices it should not be overlooked that there is positive evidence for Huhbard models being much more fond of ferromagnetism on non-bipartite lattices.15. 22 To avoid confusion in view of the absence of particle-hole symmetry for non-bipartite lattices we assume the nearest neighbor hopping amplitude $t$ to be positive (which implies the band minimum to be found at the center of the Brillouin zone). It is then demon- 


\section{Ferromagnetism in Hubbard Models}

strated with rather simple variational wave functions that for less than half-filling $(n<1)$ the Nagaoka state is never a ground state of the Hubbard model. For more than half-filling $(n>1)$ both variational estimates 15,22 and Lanczos data20 suggest sizeable regimes with a Nagaoka ground state. This is perfectly consistent with the Nagaoka theorem which predicts ferromagnetic polarons for $n>1$ only. The triangular lattice which is a prototype two-dimensional non-bipartite lattice has been investigated with particular care. The best variational calculation $\$ 23$ estimate the critical density at infinite coupling $U$ as $n_{c r} \leq 1.887$, while Lanczos data appear to confirm 20 that there is a Nagaoka ground state for $1<n<1.76$. For the three-dimensional $f c c$ and $h c p$ lattices similar investigation $\$ 15,22,20$ suggest a Nagaoka ground state for the whole regime $1<n<2$ at sufficiently large coupling. The same type of behavior follows from Lanczos data for a quasi-one-dimensional lattice, the chain of triangles.20 We thus have good evidence for the rather striking phenomenon of fully polarized ground states in the limit of small hole densities (relative to complete filling, i.e. to $n=2$ ). It is this phenomenon which we are going to use in this paper as a starting point for opening a new route towards the understanding of the ferromagnetism of Hubbard models. Since one can always map the limit of small hole densities $(n \rightarrow 2)$ onto the limit of small particle densities $(n \rightarrow 0)$, employing a particle-hole transformation, we will call this route the "low density route" in contrast to what we called the "low doping route" in the discussion above.

In what follows we will consider the $t-t^{\prime}$ Hubbard chain

$$
\mathcal{H}=-\sum_{i \sigma}\left(t c_{i \sigma}^{\dagger} c_{i+1 \sigma}+t^{\prime} c_{i \sigma}^{\dagger} c_{i+2 \sigma}+\text { h.c. }\right)+U \sum_{i} n_{i \uparrow} n_{i \downarrow} .
$$

For the special choice $t^{\prime}=t$ this model describes the chain of triangles mentioned above. We do prefer, in fact, to visualize this system as a zigzag chain of atoms rather than as a linear chain because we will have to satisfy the condition $t^{\prime}>t / 4$ to obtain a ferromagnetic ground state. This condition can probably only be met if the second neighbor distance is shorter than twice the first neighbor distance. With this in mind we also avoid any conceptual conflict with the "no-go-theorem" for ferromagnetism in strictly one-dimensional systems.24

As we want to focus on the low density limit of the zigzag chain we imagine that a particle-hole transformation and a gauge transformation have been performed such that from now on we will be interested in the case $t>0$ and $t^{\prime}<0$. Looking at the dispersion

$$
\epsilon(k)=-2 t \cos k-2 t^{\prime} \cos 2 k
$$

of the $t-t^{\prime}$ band we observe a qualitative change at the bottom of the band as $t^{\prime}$ decreases: the band minimum at $k=0$ splits into two equivalent minima for $t^{\prime}<-t / 4$. We will see that in the low density limit the existence of these two band minima is responsible for the formation of a Nagaoka ground state in the strong coupling limit.

The two minima form around momenta $\pm k_{0}$ where $\cos k_{0}=-t / 4 t^{\prime}$. At low densities only states in the neighborhood of these two minima matter. It is important for the following argument that due to the ultra-violet stability of onedimensional systems which implies intrinsic high energy cut-offs this is also true for 


\section{E. Müller-Hartmann}

the interacting system. In the presence of two band minima the system therefore develops an internal degree of freedom (left valley - right valley) at low densities. Introducing the notation $\tilde{c}_{k \tau \sigma}^{\dagger}:=c_{k-\tau k_{0}, \sigma}^{\dagger}(\tau= \pm 1)$ and fitting cosine bands $\tilde{\epsilon}_{\tau}(k)=\tilde{\epsilon}-2 \tilde{t} \cos \left(k-\tau k_{0}\right)$ with $\tilde{\epsilon}=2 t^{\prime}+t^{2} / 4 t^{\prime}$ and $\tilde{t}=-4 t^{\prime}\left[1-\left(t / 4 t^{\prime}\right)^{2}\right]$ into the two minima of the original dispersion (2) we express the original Hamiltonian $\mathcal{H}$ in terms of new fields distinguished by the symbol ${ }^{\sim}$. The result of this simple change of notation will be discussed using the new Hamiltonian

$$
\begin{aligned}
\tilde{\mathcal{H}}= & \tilde{\epsilon} N-\tilde{t} \sum_{i \tau \sigma}\left(\tilde{c}_{i \tau \sigma}^{\dagger} \tilde{c}_{i+1 \tau \sigma}+\text { h.c. }\right)+U \sum_{i \tau} \tilde{n}_{i \tau \uparrow} \tilde{n}_{i \tau \downarrow} \\
& -\tilde{J} \sum_{i}\left(\tilde{\mathbf{S}}_{i+} \cdot \tilde{\mathbf{S}}_{i-}-\frac{1}{4} \tilde{n}_{i+} \tilde{n}_{i-}\right) .
\end{aligned}
$$

where $\tilde{\mathbf{S}}_{i \tau}$ and $\tilde{n}_{i \tau}$ denote the spin and particle density operators of state $\tau= \pm$ at site $i$.

The Hamiltonian $\tilde{\mathcal{H}}$ describes two Hubbard chains with nearest neighbor hopping $\tilde{t}$ which are coupled to each other by the on-site exchange interaction $\tilde{J}$. It has twice as many degrees of freedom as the original Hamiltonian (1). Nevertheless, $\mathcal{H}$ and $\tilde{\mathcal{H}}$ are closely related in the limit of small particle densities: for $\tilde{J}=2 U$ the two models have one and the same continuum limit. The ferromagnetic exchange interaction of strength $2 U$ results from the inter-valley scattering contained in the Hubbard term of the original model.

The presence of a ferromagnetic exchange interaction $\tilde{J}>0$ in $\tilde{\mathcal{H}}$ suggests to expect ferromagnetic ground states for this model. It is, in fact, possible to arrive at the following rigorous statement:

- At $U=\infty$, for any $\tilde{J}>0$ and for any particle number $0<N<2 L$ all the ground states of $\tilde{\mathcal{H}}$ have maximum total spin $S=N / 2$.

Here $L$ is the number of sites per chain such that $N=2 L$ corresponds to halffilling of the double chain system. The proof of the above statement is quite simple. Let us first consider the case $\tilde{J}=0$ where we have two uncoupled Hubbard chains. From the exact solution 13 we know that at $U=\infty$ the ground states are given by a Slater determinant of spinless particles for each chain combined with a completely arbitrary spin wave function. Since the kinetic energy is a convex function of the particle number the particles distribute equally among the two chains $\left(N_{+}=N_{-}\right.$ for even $N$ and $N_{+}=N_{-} \pm 1$ for odd $N$ ). Including now the exchange term we see that the energy of the ground states is increased unless particles are in a triplet state whenever they meet at the same site. The only way to make sure that particles never meet in a singlet state is to have maximum total spin. This simple argument is easily made rigorous by a formal proof that out of the subspace of completely spin degenerate ground states of the uncoupled chains only those with a totally symmetric spin wave function are uneffected by the positive semi-definite exchange term.

The proof that $\tilde{\mathcal{H}}$ has no other than fully polarized ground states is an interesting result in its own right. Interpreting the internal degree of freedom as an orbital degeneracy we can rephrase this result by saying that a Hubbard chain with 


\section{Ferromagnetism in Hubbard Models}

orbital degeneracy and first Hund's rule coupling has Nagaoka ground states for $U=\infty$. We are reminded of the fact that orbital degeneracy combined with ferromagnetic Hund's rule coupling is a genuine feature of real ferromagnets in the first place. Probably, omitting orbitaldegeneracy in modeling metallic ferromagnets by the single band Hubbard modell 10 w was an oversimplification after all. In cases where the Hubbard interaction alone is not sufficient to drive a system ferromagnetic Hund's rule coupling might serve to provide the final kick.

Despite of this remark we now turn back to the original single band model. Due to the equivalence of models discussed above we conclude that the continuum limit of $\mathcal{H}$ has only fully polarized ground states at $t^{\prime}<-t / 4$ and $U=\infty$. This result represents the first proof of metallic ferromagnetism in a Hubbard model. Applying a scale transformation to the continuum model we find the following formula for the ground state energy per site at given densities of particles $n$ and magnetization $m$ :

$$
E_{0} / L=(\tilde{\epsilon}-2 \tilde{t}) n+n^{3} \tilde{t} \Phi(U / n \tilde{t}, m / n) .
$$

We don't know the scaling function $\Phi(u, \mu)$ since the model is not integrable. But from the above proof we infer

$$
\Phi(\infty, \mu)>\Phi(\infty, 1) \quad(\mu<1) .
$$

The above result opens a low density route towards the understanding of the ferromagnetism of Hubbard models on non-bipartite lattices. We have learned that in the presence of several valleys at the bottom (or top, after a particlehole transformation) of the band the Hubbard interaction implies a ferromagnetic

exchange interaction between particles in the various valleys. In one dimension this turned out to be sufficient for making the strong coupling system ferromagnetic. It is presently not obvious how this result can be generalized. Two valleys are also found ip the band structure of the triangular lattice. The discussion presented above tells us 23 that in this case two valleys are not sufficient for ferromagnetism at low densities. Nevertheless, multi-valley situations seem to be a generic prerequisite for Hubbard models having ferromagnetic ground states in a broad range of particle densities. One may wonder whether the low density route towards ferromagnetism will turn out to be more successful than the low doping route.

\section{ACKNOWLEDGMENTS}

This research is supported by SFB 341 of Deutsche Forschungsgemeinschaft.

\section{REFERENCES}

1. M.C. Gutzwiller, Phys. Rev. Letters 10, 159 (1963).

2. J. Hubbard, Proc. Roy. Soc. London A 276, 238 (1964).

3. J. Kanamori, Prog. Theor. Phys. 30, 275 (1964).

4. H. Lieb, Phys. Rev. Letters 62, 1201 (1989).

5. A. Mielke and H. Tasaki, Commun. Math. Phys. 158, 341 (1993).

6. R. Strack and D. Vollhardt, Phys. Rev. Letters 72, 3425 (1994).

7. H. Tasaki, Phys. Rev. Letters 73, 1158 (1994). 


\section{E. Müller-Hartmann}

8. Y. Nagaoka, Solid State Comm. 3, 409 (1965) and Phys. Rev. 147, 392 (1966).

9. D.J. Thouless, Proc. Phys. Soc. London 86, 893 (1965).

10. D.C. Mattis, "The Theory of Magnetism I", Springer Series in Solid State Sciences 17, Springer, Berlin-Heidelberg-New York 1981.

11. H. Tasaki, Phys. Rev. B 40, 9192 (1989).

12. M. Takahashi, J. Phys. Soc. Japan 51, 3475 (1982); Y. Fang et al., Phys. Rev. B 40, 7406 (1989); K. Hashimoto, J. Phys. Soc. Japan 54, 33 (1985) and A. Barbieri et al., Phys. Rev. B 41, 11697 (1990).

13. E.H. Lieb and F.Y. Wu, Phys. Rev. Letters 20, 1445 (1968).

14. P. Fazekas, B. Menge and E. Müller-Hartmann, Z. Physik B 78, 69 (1990).

15. B.S. Shastry, H.R. Krishnamurthy and P.W. Anderson, Phys. Rev. B 41, 2375 (1990).

16. A.G. Basile and V. Elser, Phys. Rev. B 41, 4842 (1990).

17. W. von der Linden and D.M. Edwards, J. Phys. Cond. Matter 3, 4917 (1991).

18. Th. Hanisch and E. Müller-Hartmann, Ann. Physik 2, 381 (1993).

19. W.O. Putikka, M.U. Luchini and M. Ogata, Phys. Rev. Letters 69, 2288 (1992).

20. R. Hirsch, "Zum Magnetismus stark korrelierter Fermionensysteme", Dissertation, University of Cologne (1994) and Verlag Shaker, Aachen 1994.

21. S. Liang and H. Pang, preprint.

22. E. Müller-Hartmann, Th. Hanisch and R. Hirsch, Physica B 186-188, 834 (1993).

23. A. Ritzl, Diplomarbeit, University of Cologne (1994); Th. Hanisch, B. Kleine, A. Ritzl and E. Müller-Hartmann, to be published.

24. E. Lieb and D. Mattis, Phys. Rev. 125, 164 (1962). 